\title{
Relationship among the leptin-to-adiponectin ratio, systemic inflammation, and anisocytosis: a plausible pathophysiological mechanism of a novel cardiovascular risk marker
}

\author{
Patricio López-Jaramillo', Diego Gómez-Arbeláez ${ }^{1,2}$, Jose López-López ${ }^{1,3}$ \\ 1 MASIRA Institute, Faculty of Health Sciences, University of Santander (UDES), Bucaramanga, Colombia \\ 2 Division of Vascular Surgery, Hospital 12 de Octubre, Madrid, Spain \\ 3 Center for Cardiometabolic Diseases Prevention (CIPCA), Floridablanca, Colombia
}

RELATED ARTICLE by Rostoff et al, see p. 420
Correspondence to: Patricio López-Jaramillo, MD, PhD, FACP, MASIRA Institute, Faculty of Health Sciences, University of Santander (UDES), Campus Universitario Lagos del Cacique, Calle 70 № 55-210, 680003 Bucaramanga, Colombia, phone: +573153068939, email: jplopezj@gmail.com Received: April 8, 2020. Accepted: April 9, 2020. Published online: May 25, 2020. Kardiol Pol. 2020; 78 (5): 381-382 doi:10.33963/KP.15381 Copyright by the Author(s), 2020
Anisocytosis, which is determined by red blood cell distribution width (RDW), indicates the presence of erythrocytes of widely differing volumes and is mainly employed for differential diagnosis of anemias. ${ }^{1}$ However, it has been suggested that anisocytosis (ie, high RDW) is associated with the severity and prognosis of several diseases, including type 2 diabetes (T2D) and cardiovascular diseases (CVDs)., ${ }^{1,2}$ Furthermore, studies evaluating its diagnostic accuracy have reported good results. In a cohort of 3529 patients, the RDW value independently predicted long-term all-cause mortality and cardiovascular comorbidities such as hypertension, T2D, peripheral artery disease, and ischemic cardiac disease. ${ }^{3}$ However, to date, it remains to be established by which mechanism increased RDW is associated with cardiovascular risk or whether it is actually a surrogate marker of an underlying metabolic disorder.

On the other hand, the leptin-to-adiponectin ratio and low-grade systemic inflammation have a well-established role in the pathogenesis of T2D and CVD. Leptin and adiponectin have opposing effects on systemic low-grade inflammation and insulin resistance. ${ }^{4,5}$ Leptin upregulates proinflammatory and atherogenic cytokines, such as tumor necrosis factor $\alpha$ and interleukin 6 , while adiponectin downregulates the expression and release of several proinflammatory mediators and has anti-inflammatory, antiatherogenic, and antidiabetic properties..$^{4,5}$
Leptin is mainly produced by mature adipocytes and plays a key role in the long-term regulation of body energy balance and body weight. Paradoxically, leptin levels are significantly increased in subjects with obesity, suggesting leptin resistance when produced excessively..$^{4-6}$ Meanwhile, plasma adiponectin levels are inversely related to the amount of visceral fat. ${ }^{7}$ Therefore, in patients with obesity and cardiometabolic diseases, leptin levels are elevated, whereas adiponectin levels are decreased. This leptin-to-adiponectin imbalance leads to a proinflammatory state, which is associated with insulin resistance and endothelial dysfunction, promoting the loss of the vasodilatory, antithrombotic, and antiatherogenic properties of the endothelial cells. ${ }^{4,5,8}$

In a previous study on an ex vivo model in which fragments of internal mammary arteries were obtained from patients undergoing coronary artery bypass graft surgery, abdominal obesity was correlated with leptin-to-adiponectin imbalance and was associated with a weaker vascular response to acetylcholine and an increased vasoconstrictor response to angiotensin II. ${ }^{9}$ Moreover, leptin and adiponectin have been shown to be independently associated with T2D and CVD, but the association with the leptin-to-adiponectin ratio is stronger than with leptin or adiponectin levels separately. ${ }^{4,9,10}$ Hence, the serum leptin-to-adiponectin ratio may be used as an integrated marker 
of adiposity, insulin resistance, and vascular dysfunction, which can be useful for cardiovascular risk stratification in clinical practice. ${ }^{4}$

In the current issue of Kardiologia Polska (Kardiol Pol, Polish Heart Journal), Rostoff et $\mathrm{al}^{11}$ published an interesting study, in which they evaluated the relationship between the leptin-to-adiponectin ratio, systemic low-grade inflammation, and RDW in optimally treated patients with T2D and established atherosclerotic CVD. The study demonstrates a positive association between RDW and leptin-to-adiponectin imbalance. Furthermore, they reported that RDW was positively correlated with several inflammatory markers, including tumor necrosis factor $\alpha(\mathrm{TNF}-\alpha)$ and soluble intercellular adhesion molecule 1 (sICAM-1).

Certainly, these results help to understand the mechanisms underlying increased RDW values (ie, anisocytosis) in patients with T2D and CVD. Given the reported data, it seems reasonable to suggest that anisocytosis could reflect an underlying state of leptin-to-adiponectin imbalance, inflammation, and insulin resistance. This would explain its good performance as a cardiometabolic risk marker. Several metabolic and inflammatory pathways may be involved in the alteration of erythropoiesis and the consequent appearance of anisocytosis, caused by adipokines. ${ }^{12-14}$

As mentioned, leptin-to-adiponectin imbalance induces a proinflammatory state, and inflammation is probably the main mechanism responsible for anisocytosis in patients with cardiometabolic diseases, as several proinflammatory cytokines inhibit synthesis or activity of erythropoietin. ${ }^{12}$ Inflammation might also promote a shortened erythrocyte half-life, inhibition of erythroid cell differentiation, and alterations of iron metabolism resulting in iron-restricted erythropoiesis..$^{13}$ Leptin is implicated in the proliferation of erythroid precursor cells, stimulation of erythrocyte lineage cell differentiation, and induction of hepcidin expression, the main regulator of iron availability. ${ }^{14}$ Nonetheless, future research is required to fully elucidate the potential mechanisms by which anisocytosis is associated with leptin-toadiponectin imbalance and low-grade inflammation. Based on current evidence, it seems coherent to conclude that the determination of RDW has a much broader application than the simple differential diagnosis of anemia and it can also be considered a useful and plausible cardiovascular risk marker.

\section{ARTICLE INFORMATION}

DISCLAIMER The opinions expressed by the author are not necessarily those of the journal editors, Polish Cardiac Society, or publisher.

CONFLICT OF INTEREST None declared.

OPEN ACCESS This is an Open Access article distributed under the terms of the Creative Commons Attribution-NonCommercial-NoDerivatives 4.0 International License (CC BY-NC-ND 4.0), allowing third parties to download articles and share them with others, provided the original work is properly cited, not changed in any way, distributed under the same license, and used for noncommercial purposes only. For commercial use, please contact the journal office at kardiologiapolska@ptkardio.pl.

HOW TO CITE López-Jaramillo P, Gómez-Arbeláez D, López-López J. Relationship among the leptin-to-adiponectin ratio, systemic inflammation, and anisocytosis: a plausible pathophysiological mechanism of a novel cardiovascular risk marker. Kardiol Pol. 2020; 78: 381-382. doi:10.33963/KP.15381

\section{REFERENCES}

1 Salvagno GL, Sanchis-Gomar F, Picanza A, Lippi G. Red blood cell distribution width: a simple parameter with multiple clinical applications. Crit Rev Clin Lab Sci. 2015; 52: 86-105.

2 Borné Y, Smith JG, Melander 0, Engström G. Red cell distribution width in relation to incidence of coronary events and case fatality rates: a population-based cohort study. Heart. 2014; 100: 1119-1124.

3 Laufer Perl M, Havakuk 0, Finkelstein A, et al. High red blood cell distribution width is associated with the metabolic syndrome. Clin Hemorheol Microcirc. 2015; 63: 35-43.

4 López-Jaramillo P, Gómez-Arbeláez D, López-López J, et al. The role of leptin/ adiponectin ratio in metabolic syndrome and diabetes. Horm Mol Biol Clin Investig. 2014; 18: 37-45.

5 Galic S, Oakhill JS, Steinberg GR. Adipose tissue as an endocrine organ. Mol Cell Endocrinol. 2010; 316: 129-139.

6 Stefanović A, Kotur-Stevuljević J, Spasić S, et al. The influence of obesity on the oxidative stress status and the concentration of leptin in type 2 diabetes mellitus patients. Diabetes Res Clin Pract. 2008; 79: 156-163.

7 Okauchi Y, Kishida K, Funahashi T, et al. Changes in serum adiponectin concentrations correlate with changes in BMI, waist circumference, and estimated visceral fat area in middle-aged general population. Diabetes Care. 2009; 32: e122.

8 Lopez-Jaramillo P, Casas JP. Endothelial dysfunction, angiotensin-converting enzyme inhibitors and calcium antagonists. J Hum Hypertens. 2002; 16 (suppl 1): S34-S37.

9 Rueda-Clausen CF, Lahera V, Calderon J, et al. The presence of abdominal obesity is associated with changes in vascular function independently of other cardiovascular risk factors. Int J Cardiol. 2010; 139: 32-41.

10 Oda N, Imamura S, Fujita T, et al. The ratio of leptin to adiponectin can be used as an index of insulin resistance. Metabolism. 2008; 57: 268-273.

11 Rostoff $P$, Siniarski A, Haberka M, et al. Relationship among the leptin-to-adiponectin ratio, systemic inflammation, and anisocytosis in well-controlled type 2 diabetic patients with atherosclerotic cardiovascular disease. Kardiol Pol. 2020; 78: $420-428$.

12 Jelkmann W. Proinflammatory cytokines lowering erythropoietin production. J Interferon Cytokine Res. 1998; 18: 555-559.

13 Weiss G, Ganz T, Goodnough LT. Anemia of inflammation. Blood. 2019; 133: 40-50.

14 Coimbra S, Catarino C, Santos-Silva A. The role of adipocytes in the modulation of iron metabolism in obesity. Obes Rev. 2013; 14: 771-779. 